Regular paper

\title{
Traveling-wave antenna based on metamaterial transmission line structure for use in multiple wireless communication applications
}

\author{
Mohammad Alibakhshi-Kenari ${ }^{a}$, Mohammad Naser-Moghadasi a,*, R. A. Sadeghzadeh ${ }^{\text {b }}$, \\ Bal S. Virdee ${ }^{\mathrm{c}}$, Ernesto Limiti ${ }^{\mathrm{d}}$ \\ a Faculty of Eng., Science and Research Branch, Islamic Azad University, Tehran, Iran \\ ${ }^{\mathrm{b}}$ Faculty of Electrical Eng., K. N. Toosi University of Technology, Tehran, Iran \\ ' London Metropolitan University, Center for Communications Technology, Faculty of Life Sciences and Computing, London N7 8DB, UK \\ ${ }^{\mathrm{d}}$ Dipartimento di Ingegneria Elettronica, Università degli Studi di Roma Tor Vergata, Via del Politecnico 1, 00133 Roma, Italy
}

\section{A R T I C L E I N F O}

Article history:

Received 27 February 2016

Accepted 4 October 2016

\section{Keywords:}

Traveling-wave antenna (TWA)

Metamaterial (MTM)

X-shaped slot

Broadband antenna

\begin{abstract}
A B S T R A C T
This paper introduces a left-handed metamaterial traveling-wave antenna (TWA) based on metamaterial transmission-line structure to enhance the gain and radiation efficiency of the antenna without trading on its fractional bandwidth. The antenna consists of a series of coupled unit-cells comprising "X-shaped" slots which are inductively terminated to ground. Effective aperture of the antenna can be increased by increasing the number of unit-cells. The consequence of this is enhanced gain and radiation efficiency performance with no adverse affect on its fractional bandwidth. The antenna's characterizing parameters were extracted using 3D electromagnetic simulation tool $\left(\mathrm{HFSS}^{\mathrm{TM}}\right)$, and the antenna was fabricated using standard PCB manufacturing techniques on a $1.6 \mathrm{~mm}$ thick dielectric substrate with permittivity of 2.2. The antenna operates from $0.4 \mathrm{GHz}$ to $4.7 \mathrm{GHz}$. The antenna has an electrical size of $0.017 \lambda_{0} \times 0.006 \lambda_{0}$ $\times 0.002 \lambda_{0}$, where $\lambda_{0}$ is free space wavelength at $400 \mathrm{MHz}$. The proposed antenna is significantly smaller than its conventional counterparts. Antenna's measured optimum gain and radiation efficiency are $2 \mathrm{dBi}$ and $65 \%$, respectively, at $2.5 \mathrm{GHz}$. These features make the antenna attractive for use in multiple wireless communication applications.
\end{abstract}

(c) 2016 Elsevier GmbH. All rights reserved.

\section{Introduction}

Printed planar antennas are very popular as they are low profile, light weight, and consist of a metal layer separated from the ground plane by a dielectric substrate $[1,2]$. Advantages of printed planar antennas are that they radiate energy with moderately high gain in a direction perpendicular to the substrate and can be fabricated using low cost printed circuit board techniques [3-5]. The basic operating principle of a printed antenna is that the space between the metal patch and ground plane acts like a section of parallel plate waveguide. Neglecting radiation loss, the edge of patch is an open circuit, so that energy reflects and remains contained between the patch and the ground plane to create a high$\mathrm{Q}$ factor device with a narrow bandwidth. Hence, patch antennas have limited bandwidth; this means that the specified input impedance of the antenna is maintained only over a small range around

\footnotetext{
* Corresponding author.

E-mail addresses: makenari@mtu.edu (M. Alibakhshi-Kenari),mn.moghaddasi@ srbiau.ac.ir (M. Naser-Moghadasi), sadeghz@eetd.kntu.ac.ir (R.A. Sadeghzadeh), b.virdee@londonmet.ac.uk (B.S. Virdee), limiti@ing.uniroma2.it (E. Limiti).
}

its central operating frequency. To overcome this challenge several metamaterial (MTM) structures like Split Ring Resonator (SRR) [6], Spiral, Rod, Omega, S-shape, Symmetric Rings etc. have already been explored by previous researchers $[7,8]$.

This paper introduces a new and distinct metamaterial unit-cell which is used to implement a traveling-wave planar antenna structure referred to here as "X-shaped" antenna. The proposed antenna is based on metamaterial unit cells created using X-shaped slots which are inductively terminated to the ground plane with a metal via-hole. The structure exhibits negative permittivity and permeability over a specific frequency range and negative refractive index. By coupling together a number of these unit-cells in series we can increase the aperture of the antenna thereby increasing its gain and radiation efficiency without adversely affecting the fractional bandwidth of the antenna. This property is contrary to that observed in conventional antennas.

\section{Design methodology}

The proposed traveling wave antenna, shown in Fig. 1, consists of a rectangular radiation patch on which are constructed four 


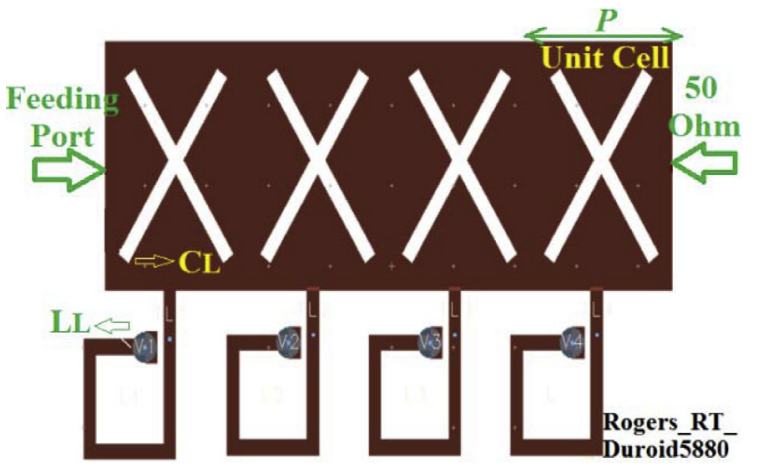

(a)

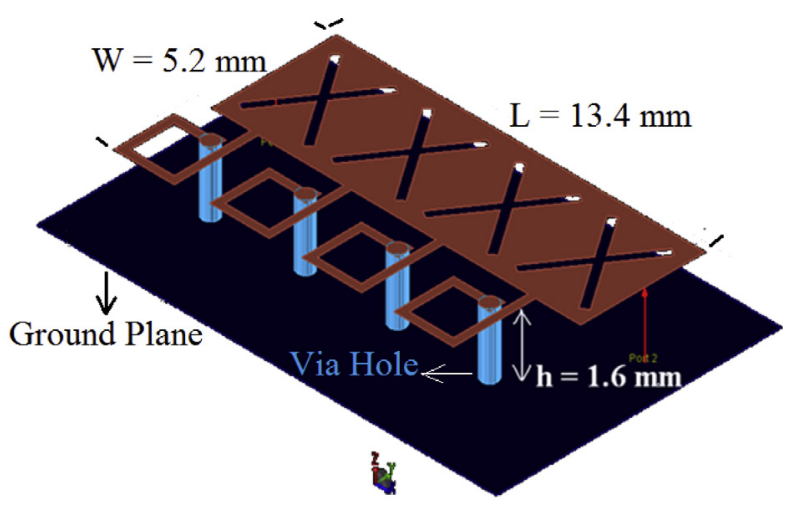

(b)

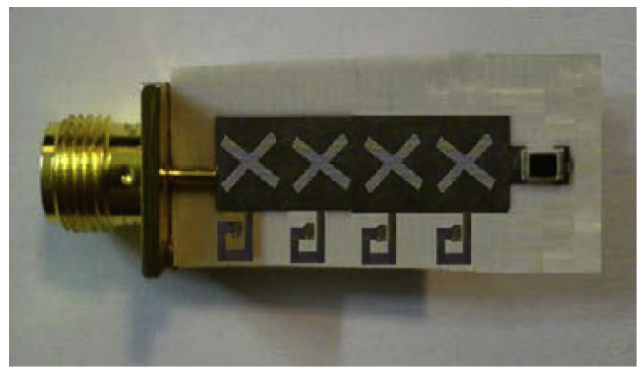

(c)

Fig. 1. Antenna layout, (a) top layer, (b) isometric view of simulation model, and (c) fabricated prototype.

unit-cells comprising the X-shaped slot which is terminated to ground through an inductive stub. The antenna was designed and constructed on Rogers RT/Duroid5880 (low loss) substrate with $\tan \delta=0.0009$, dielectric constant $\left(\varepsilon_{r}\right)$ of 2.2 and height $(h)$ of $1.6 \mathrm{~mm}$. Two waveguide ports are defined at the left and righthand side, as shown in Fig. 1(a), where port-1 is excited by the input signal, and port-2 is matched to $50 \mathrm{ohm}$ load using SMD1206 that is terminated to ground. The proposed antenna configuration provides a guiding structure for a traveling wave as the main radiating mechanism. Here the surface currents that generates the RF signal travels through the antenna in one direction from the input port 1 to a terminated port 2, which is in contrast to conventional standing wave or resonant antenna, such as the monopole or dipole. The results presented below show that the proposed traveling wave antenna, which is a non-resonant structure, exhibits a wider operational bandwidth.

The equivalent circuit model of the antenna unit-cell is shown in Fig. 2(a). The unit-cell essentially acts like the series lefthanded ( $\mathrm{LH})$ capacitance $\left(C_{L}\right)$, and the rectangular stub acts like a shunt LH inductor $\left(L_{L}\right)$. The shunt right-handed $(\mathrm{RH})$ capacitance $\left(C_{R}\right)$ and the series RH inductance $\left(L_{R}\right)$ represent parasitic effects which are unavoidable as they are generated by gaps between patch and ground plane due to the current flowing over the patch. Losses of the structure are modeled by $R_{R}, G_{R}, R_{L}$ and $G_{L} . R_{R}$ and $G_{R}$ are RH losses and $R_{L}$ and $G_{L}$ are LH losses. Essential characteristics of a CRLH unit cell can be inferred from analysis of the loss-less equivalent circuit as described in [8]. At low frequencies, $L_{R}$ and $C_{R}$ tend to be short and open, respectively, so that the equivalent circuit is essentially reduced to the series- $C_{L} /$ shunt- $L_{L}$ circuit, which is left-handed since it has antiparallel phase and group velocities; this LH circuit is of highpass nature; therefore, below a certain cutoff, a LH stopband is present. At high frequencies, $C_{L}$ and $L_{L}$ tend to be short and open, respectively, so that the equivalent circuit is essentially reduced to the series $-L_{R} /$ shunt- $C_{R}$ circuit, which is right-handed since it has parallel phase and group velocities; this LH circuit is of lowpass nature; therefore, above a certain cutoff, a RH stopband is present. The analysis in [8] shows that the left-handed structure exhibits negative permeability and permittivity. Unlike conventional or normal right right-handed unit cell based structures, the distinguishing feature of a lefthanded structure is its dimensions; in particular, its length is independent of guided wavelength, which makes the structure significantly smaller. Dispersion diagram of the CRLH unit cell structure shown in Fig. 2(b) was determined by substituting the $\mathrm{S}$ parameters, which were obtained with $\mathrm{HFSS}^{\mathrm{TM}}$, in Eq. (1) as described in [8]:

$\beta=\cos ^{-1}\left(\frac{1-S_{11} S_{12}+S_{12} S_{21}}{2 S_{21}}\right) / l$

The dispersion diagram shows the range of LH characteristics $(0.65-2.25 \mathrm{GHz})$ and the range of $\mathrm{RH}$ characteristics (2.25$4.4 \mathrm{GHz}$ ). The slow-wave and fast-wave regions have been indicated in Fig. 2(b). The expression of Air-line, which demarcates the fast-wave $\left(b<K_{o}\right)$ and slow-wave $\left(b>K_{o}\right)$ regions (where $K_{o}$ is the free space wavenumber) is given by Eq. (2),

Air-line $=\frac{\omega p}{c}$

where $p$ is the period of the unit-cell as shown in Fig. 1, and $c$ is the speed of light.

The characterizing parameters of the antenna structure are: $L=13.4 \mathrm{~mm}, W=5.2 \mathrm{~mm}, h=1.6 \mathrm{~mm}, L_{R}=3.25 \mathrm{nH}, C_{L}=4.1 \mathrm{pF}$, $C_{R}=1.3 \mathrm{pF}$ and $L_{L}=5.2 \mathrm{nH}$, which were extracted from the simulation model using HFSS $^{\mathrm{TM}}$. The prototype antenna, shown in Fig. 1(c), was fabricated using standard manufacturing techniques. Each of the unit-cell occupies an area of $2.5 \times 5.2 \mathrm{~mm}^{2}$ $\left(0.003 \lambda_{0} \times 0.006 \lambda_{0}\right.$, where $\lambda_{0}$ is free space wavelength at $400 \mathrm{MHz})$. Considering the size of SMD $(3.4 \mathrm{~mm})$, the overall size of the antenna is $13.4 \times 5.2 \times 1.6 \mathrm{~mm}^{3}$ or $0.017 \lambda_{0} \times 0.006 \lambda_{0} \times$ $0.002 \lambda_{0}$. 


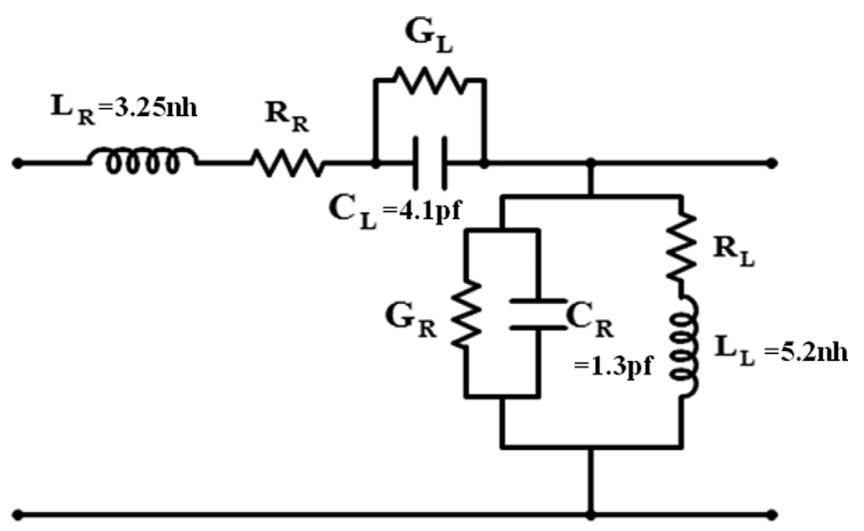

(a)

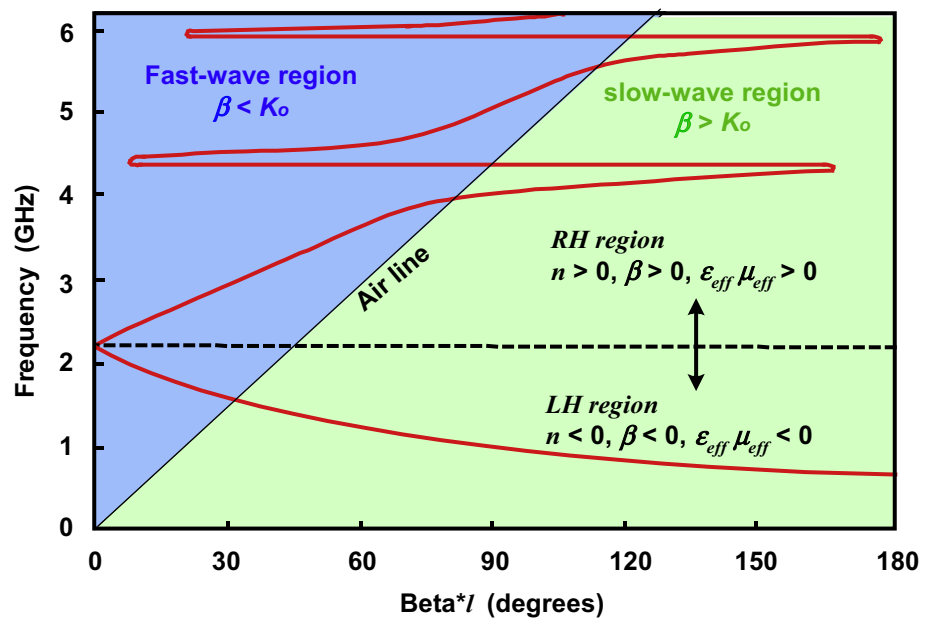

(b)

Fig. 2. (a) Equivalent circuit model of the unit-cell, and (b) the dispersion diagram of the loss-less CRLH unit-cell.

Antennas that operate in a resonance configuration have limited bandwidth due to the destructive interference of the waves. Bandwidth can be increased by blocking the standing wave and instead using a traveling wave. The proposed antenna provides a guiding structure for a traveling wave as the main radiating mechanism. In this configuration, the voltage and current are in phase and have the same $\mathrm{e}^{-\mathrm{j} \gamma z}$ distribution along the length, where $\gamma=K z=\beta-\mathrm{j} \alpha$. The lower limit of the leaky wave bandwidth is the frequency at which $\alpha=\beta$ and the upper limit occurs when $\beta=K_{o}$. In this case the surface currents that generates the RF signal travels through the antenna in one direction from the input port 1 to a terminated port 2 , which is in contrast to conventional standing wave or resonant antenna, such as the monopole or dipole. The results presented show that the proposed traveling wave antenna, which is a non-resonant structure, exhibits a wider operational bandwidth.

The simulated and measured reflection coefficient response of the proposed TWA is shown in Fig. 3. It can be discerned from Fig. 3 that the antenna highly receptive at three distinct frequencies, i.e. $1.4,2.65$ and $3.7 \mathrm{GHz}$. The simulated bandwidth of the antenna (for $\mathrm{S}_{11}<-10 \mathrm{~dB}$ ) is $4.55 \mathrm{GHz}$ from $300 \mathrm{MHz}$ to $4.85 \mathrm{GHz}$, which corresponds to a fraction bandwidth of $176.7 \%$. Measurements show the proposed antenna operates across a frequency of $400 \mathrm{MHz}$ to $4.7 \mathrm{GHz}$ (for $\mathrm{S}_{11}<-10 \mathrm{~dB}$ ), which corresponds to a fractional bandwidth of $168.62 \%$.

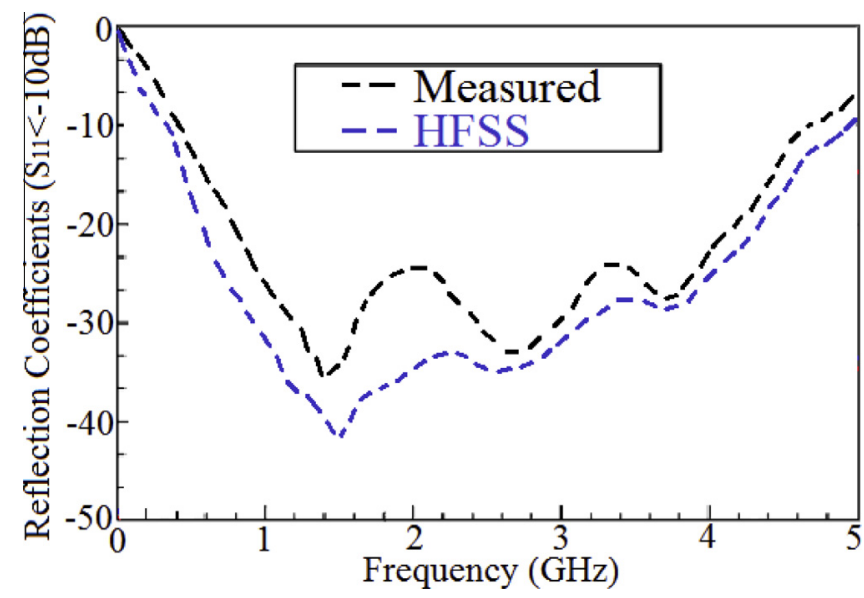

Fig. 3. Simulated and measured reflection coefficients response.

The measured radiation gain and efficiency of the proposed antenna at spot frequencies of $0.4,1.5,2.5,3.5$ and $4.7 \mathrm{GHz}$ are $0.05 \mathrm{dBi}$ and $8 \%, 1.5 \mathrm{dBi}$ and $45 \%, 2 \mathrm{dBi}$ and $65 \%, 1.5 \mathrm{dBi}$ and $42 \%$, and $0.8 \mathrm{dBi}$ and $30 \%$, respectively. The maximum gain and efficiency of $2 \mathrm{dBi}$ and 65\%, respectively, are achieved at $2.5 \mathrm{GHz}$. The measured E-plane and $\mathrm{H}$-plane radiation patterns of the 

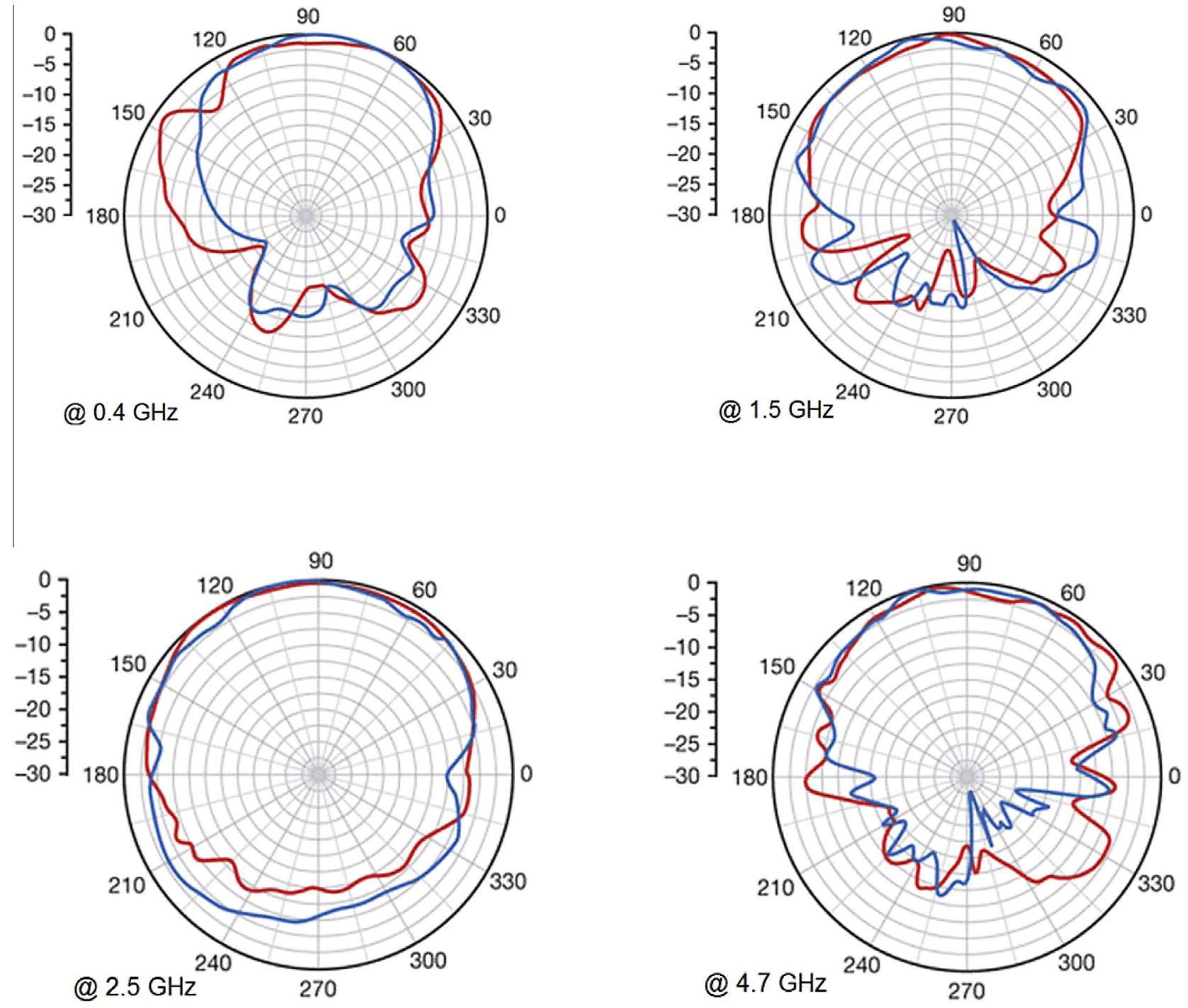

\section{- H-plane - E-plane}

Fig. 4. Measured E-plane and H-plane radiation patterns of the proposed antenna.

Table 1

Comparison of the proposed TWA with other antennas (Gain and Efficiency are optimum values).

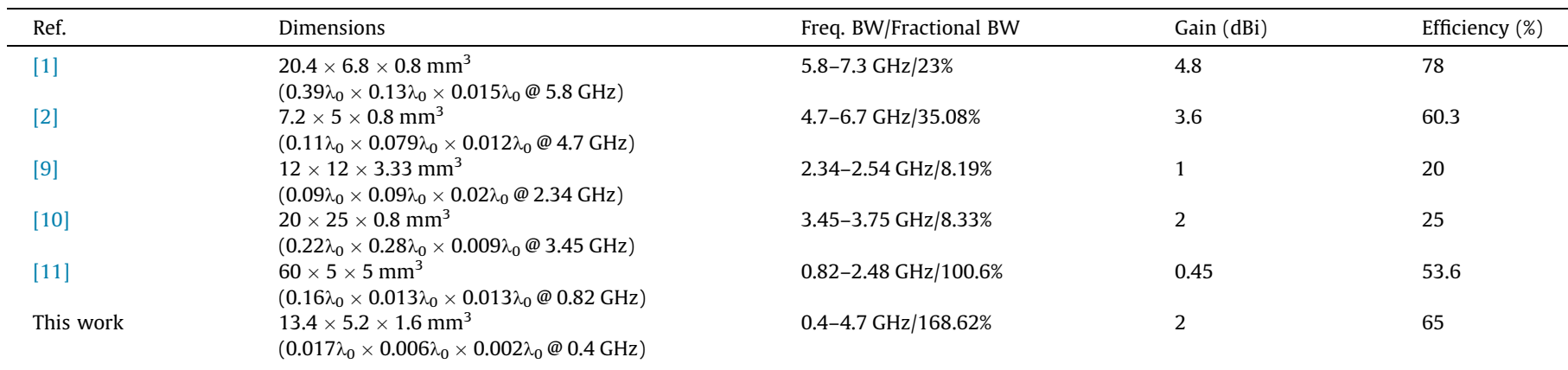

antenna at spot frequencies of $0.4,1.5,2.5$ and $4.7 \mathrm{GHz}$ are plotted in Fig. 4. It is clear from Fig. 4 that the radiation patterns of the proposed antenna have unidirectional characteristics. In addi- tion, the antenna configuration effectively increases the size of the antenna's aperture to enhance its gain and radiation characteristics. 
Compared to traditional capacitor based CRLH unit cell, the paper introduces (i) a novel method of implementing the CRLH unit cell by etching a slot in the transmission-line which is inductively shorted to ground; and (ii) the unit cell is used to realize a traveling wave radiator which is significantly shorter than a traditional traveling wave antennas as the dimensions of the antenna are independent of the wavelength. The features of the proposed antenna are: (i) compactness (with physical dimensions of $13.4 \times 5.2 \times 1.6 \mathrm{~mm}^{3}$ and electrical dimensions of $0.017 \lambda_{0}-$ $\times 0.006 \lambda_{0} \times 0.002 \lambda_{0}$ at $400 \mathrm{MHz}$ ); (ii) low profile and lightweight; (iii) broad bandwidth performance of $4.3 \mathrm{GHz}$ from 0.4 to $4.7 \mathrm{GHz}$, which corresponds to a large fractional bandwidth of $\sim 170 \%$; (iv) unidirectional radiation patterns in both $\mathrm{E}$ - and $\mathrm{H}$ - planes over the entire operating frequency band; $(v)$ and maximum gain and efficiency of $2 \mathrm{dBi}$ and $65 \%$, respectively, at $2.5 \mathrm{GHz}$. These features make the antenna attractive for use in multiple wireless communication applications.

The measured results of the proposed traveling-wave antenna are compared with other CRLH unit-cell and conventional antennas in Table 1 . These results show the operating frequency and fractional bandwidth of the proposed antenna is superior to CRLH unit-cell antennas presented in references [1] and [2]. The operating frequency is better than antennas in [1] and [2] by a factor of 2.87 and 2.15, respectively; and the fractional bandwidth is better than antennas in [1] and [2] by a factor of 7.33 and 4.8, respectively.

\section{Conclusion}

A novel traveling-wave planar antenna is presented based on metamaterial technology for enhanced gain and radiation efficiency without adversely affecting is bandwidth performance. The miniature antenna consists of coupled metamaterial unitcells comprising $\mathrm{X}$-shaped slots and shunted inductive stubs, which are implemented on a rectangular patch. The proposed antenna is easy to fabricate using conventional PCB manufacturing techniques.

\section{References}

[1] Alibakhshi-Kenari M, Naser-Moghadasi M, Virdee BS, Andújar A, Anguera J. Compact antenna based on a composite right/left handed transmission line. Microwave Opt Technol Lett 2015;57(8):1785-8.

[2] Alibakhshi Kenari M. New traveling-wave antenna resonating at $6 \mathrm{GHz}$ based on artificial transmission line metamaterial structures for RF portable devices. Open J Antennas and Propag (OJAPr) 2013;1(2). doi: http://dx.doi.org/10.4236/ ojapr.2013.12003.

[3] Collin RE. Field theory of guided waves. 2nd ed. Wiley- Interscience; 1991 chap. 12.

[4] Balanis CA. Antenna theory and design. John Wiley \& Sons; 1997.

[5] Pozar DM. Microwave and RF design of wireless systems, New York. USA: John Wiley \& Sons Inc; 2000.

[6] Alibakhshi-Kenari M, Naser-Moghadasi M, Sadeghzadah RA. Bandwidth and radiation specifications enhancement of monopole antennas loaded with split ring resonators. IET Microwaves Antennas Propag 2015;9 (14):1487-96.

[7] Engheta N, Ziolkowski RW, editors. Electromagnetic metamaterials: physics and engineering explorations. Wiley and IEEE Press; 2006.

[8] Caloz C, Itoh T. Electromagnetic metamaterials, transmission line theory and microwave applications. Wiley and IEEE Press; 2005.

[9] Lee CJ, Leong KMH, Itoh T. Broadband small antenna for portable wireless application. Int. Workshop on Antenna Technology: Small Antennas and Novel Metamaterials, iWAT 2008, March 4-6. iWAT; 2008. p. 10-3.

[10] Yu C.-C, Huang M.-H, Lin L.-K, Chang Y.-T, A compact antenna based on MTM for WiMAX, Asia-Pacific Microwave Conference, (APMC); 16-20 Dec. 2008, Macau-China.

[11] Li Y, Zhang Z, Zheng J, Feng Z. Compact heptaband reconfigurable loop antenna for mobile handset. IEEE Antennas Wirel Propag Lett 2011;10: $1162-5$.

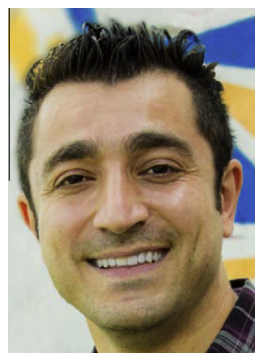

Mohammad Alibakhshi-Kenari was born in Feb. 1988 in Freiydounkenar, Mazandarn, IRAN. He received the B.Sc. (Feb. 2010) and M.Sc. (Feb. 2013) degrees in Electrical Engineering and Telecommunication from the Islamic Azad University, Najafabad Branch of Esfahan, Iran and Shahid Bahonar University of Kerman, Iran, respectively. His research interests include microwave and millimeter-wave circuits, transceivers, antennas and wave-propagation, CRLH-TLs, metamaterials, integrated RF-technologies, embedded systems, electromagnetic-waves applications and wireless telecommunication systems. The above research lines have produced more than 20 publications on refereed-international journals and presentations within international-conferences. He is now member of the Applied Computational Electromagnetics Society (ACES). He is too a reviewer for several journals including IEEE TIE, IET MAP, IEEE PTL, JIMTW, OSA, Wiley, Elsevier, Taylor \& Francis, Springer and ACES. Mr. Alibakhshi has served as a Member of the Technical Program Committee (M-TPC) at IEEE international conferences. Also, his Master Thesis entitled "Designing and Fabricating an Ultra Compact and UWB Antennas based on CRLH Metamaterial Transmission Lines with Application in Wireless Radio Transceivers" was approved and granted by Iran Telecommunication Research Center (ITRC) on December 2012 with grant number of 6987/500/T.

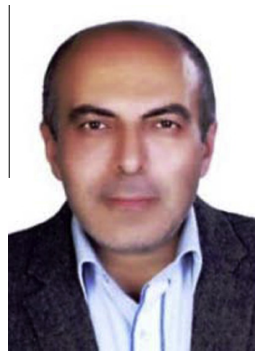

Mohammad Naser-Moghadasi was born in Iran, in 1959. He received the B.Sc. degree in Communication Eng. in 1985 from the Leeds Metropolitan University (formerly Leeds Polytechnic)-UK. Between 1985-1987 he worked as an RF-design-engineer for the Gigatech company in Newcastle Upon Tyne-UK. From 19871989, he was awarded a full scholarship by the Leeds Educational Authority to pursue an M.Phil. on Studying CAD of microwave-circuits. He received his Ph.D. in 1993, from the University of Bradford-UK. He was offered then a two-years post-doc. at the University of Nottingham-UK, to pursue research on microwave cooking of materials. From 1995, Dr. Naser-Moghadasi joined Islamic Azad University, Science and Research Branch, Iran-Tehran, where he currently is head of postgraduate studies. His main areas of interest in research are microstrip antenna, microwave passive and active circuits, RF MEMS. Dr. Naser-Moghadasi is member of the IET, MIET and IEICE. He has so far published over 150 papers.

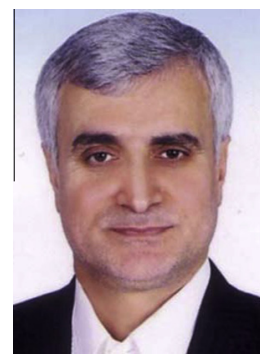

R.A. Sadeghzadeh is a full professor of communications Engineering at the faculty of Electrical Engineering of the K.N. Toosi University of Technology. He received his B.Sc. in 1984 in telecommunications Engineering from the K.N. Toosi, University of Technology in Tehran, Iran, and M.Sc. in digital Communications Engineering from the University of Bradford and UMIST (University of Manchester Institute of Science and Technology), UK as a joint program in 1987. He received his Ph.D. in electromagnetic and antenna from the University of Bradford, UK in 1990. He worked as a Post-Doctoral Research assistant in the field of propagation, electromagnetic, antenna, Bio-Medical, and Wireless communications from 1990 till 1997. From 1984 to 1985 he was with Telecommunication Company of Iran (TCI) working on Networking. Since 1997 he is with the K.N. Toosi University of Technology working with Telecommunications Dept. at the faculty of Electrical Engineering. He has published more than 175 referable papers in international journals and conferences. Professor Sadeghzadeh current interests are numerical techniques in electromagnetic, antenna, propagation, radio networks, wireless communications, nano-antennas and radar systems.

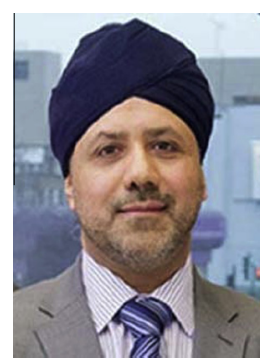

Bal Singh Virdee received the B.Sc. and MPhil degrees in Communications-Engineering from the University of Leeds-UK and his Ph.D. in Electronic-Engineering from the University of London-UK. He has worked in industry for various companies including Philips (UK) as an R\&Dengineer and Filtronic-Components Ltd. as a future products developer in the area of $\mathrm{RF} /$ microwave communications. He has taught at several academic institutions before joining London Metropolitan University where he is a Professor of Microwave-Communications in the Faculty of Life Sciences\&Computing where he Heads the Center for Communications-Technology and is the Director of London Metropolitan-Microwaves. His research, in collaboration with industry and academia, is in the area of microwave wireless communications encompassing mobile-phones to satellite-technology. Prof. Virdee has chaired 
technical sessions at IEEE international conferences and published numerous research-papers. He is Executive-Member of IET's Technical and Professional Network Committee on RF/Microwave-Technology. He is a Fellow of IET and a SeniorMember of IEEE.

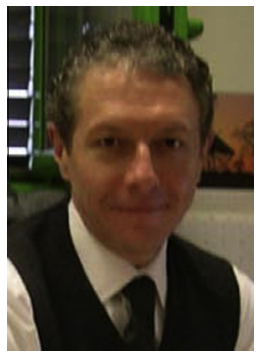

Ernesto Limiti is a full professor of Electronics in the Engineering Faculty of the University of Roma Tor Vergata since 2002, after being research and teaching assistant (since 1991) and associate professor (since 1998) in the same University. His research activity is focused on three main lines, all of them belonging to the microwave and millimetre-wave electronics area. The first one is related to characterisation and modelling for active and passive microwave and millimetre-wave devices. Regarding active devices, the research line is oriented to the small-signal, noise and large signal modelling as well as devices on diamond substrates. Novel methodologies have been developed and equivalent-circuit modelling strategies have been implemented both for small and large-signal operating regimes for different device technologies. Design methodologies and characterisation methods for low noise devices and circuits are also in focus, as well as the analysis and design methodologies for linear and nonlinear microwave circuits. The above research lines have produced more than 300 publications on refereed international journals and presentations within international conferences. Ernesto Limiti acts as a referee of international journals of the microwave and millimetre wave electronics sector and he is in the steering committee of international conferences and workshops. 